Although echocardiography was unremarkable and neither bronchial nor cardiac biopsies were performed, the presumed explanation for the symptomatology was allergic alveolitis and myocarditis. The decision not to biopsy, but to treat on empirical grounds was based on clinical judgement. The response to treatment justified the conservative management.

Numerous organ systems have been involved in clozapine-induced allergy; myocarditis (Bandelow et al, 1995), colitis (Friedberg et al, 1995), pancreatitis (Chengappa et al, 1995), hepatitis (Thatcher et al, 1995) and cutaneous reactions (Stoppe et al, 1992) have been reported. Those reactions are invariably associated with eosinophilia.

To date, only one case of clozapineinduced allergic asthma has been reported (Stoppe et al, 1992). This case suggests that a separate entity be recognised: that of clozapine-induced allergic alveolitis. In this case the patient's symptoms showed rapid progression.

Pyrexia, although frequently a benign side-effect of clozapine, in fact, indicates the need for thorough investigation and clinical vigilance. The possibility of potentially lethal cardio-respiratory complications should be considered early, especially where there is eosinophilia, and a chest X-ray, electrocardiogram, erythrocyte sedimentation rate and echocardiograph may be regarded as necessary investigations for pyrexia.

The diligent reporting of such cases may assist in the identification of predictors of such potentially lethal allergic compilations, for example age, history of smoking, length of clozapine treatment and previous cardiorespiratory disease.

In terms of progress, the pyrexia abated two days after the cessation of clozapine. A chest X-ray performed five days after discontinuation of the clozapine showed complete resolution of the interstitial shadowing. The erythrocyte sedimentation rate dropped to 42 . The patient showed dramatic clinical improvement.

Bandelow, B., Degner, D., Kreusch, U., et al (1995) Myocarditis under therapy with clozapine. Schizophrenio Research, 17. 293-294.

Chengappa, K. N. R., Polucia, M., Baker, R. W., et al (1995) Recurrent pancreatitis on clozapine re-challenge journal of Psychopharmacology, 9. 381-382.

Friodberg, J. W., Frankenburg, F. R., Burk, J., et al (1993) Clozapine - caused eosinophilic colitis. Annols of Clinical Psychiatry, 7, 97-98.
Stoppe, G., Muller, P., Fuchs, T., ot ol (1992) Lifethreatening allergic reactions to clozapine. British fournal of Psychiatry, 16, 259-261.

Thatcher, G.W., Cates, M. \& Bair, B. (1995) Clozapine induced toxic hepatitis. American Journal of Psychiatry. I52. 296-297.

T. B. Benning CJ Cummins Unit, Royal North Shore Hospital, St Leonards, Sydney, NSW 2065, Australia

\section{Prolonged bradycardia complicates antidepressive treatment with venlafaxine and ECT}

Sir: We describe the case of a patient given venlafaxine and electroconvulsive therapy (ECT), where cardiovascular complications led to interruption of the ECT.

A 42-year-old man with a severe depressive disorder and suicidal ideation failed to improve despite venlafaxine ( $300 \mathrm{~g} /$ day) combined with flurazepam (15 g/day) over 44 days. Thoracic X-radiography, electroencephalogram (EEG), computed tomography, laboratory testing and serial electrocardiogram (ECG) recordings were all normal during venlafaxine treatment and before ECT. On the 45th day venlafaxine was reduced to $150 \mathrm{~g} / \mathrm{day}$ (flurazepam unchanged). On the 46th day, $0.75 \mathrm{~g}$ atropine, $100 \mathrm{~g}$ propofol, $0.5 \mathrm{~g}$ norcurone and $50 \mathrm{~g}$ succinylcholine were given immediately before ECT (ThymatronDG, bilateral brief pulse, $100.8 \mathrm{mC}$ ). A generalised cramp was induced lasting 40 seconds. A rapid reduction in heart rate was followed by an asystole. After another atropine dose $(0.5 \mathrm{~g})$, a bradyarrhythmia (22-40 bpm) developed (for about 90 seconds) which spontaneously led to a bradycardial sinus rhythm. During three hours a normal sinus rhythm returned. Depressive symptoms completely receded with hypomania lasting about 16 hours; afterwards the depressive profile returned to its full extent. The cardiovascular events observed prompted further tests (echocardiography, 24-hour recording of ECG and blood pressure, stress-ECG) which produced normal results. Therapy administered during this phase (lithium, moclobemide, lorazepam) was unsuccessful. Six weeks later a second ECT course (seven rightside unilateral, one bilateral; 100.8$201.6 \mathrm{mC}$ ) did not produce cardiovascular complications. All psychopharmaceuticals except lorazepam had been discontinued
14 days before and the same pre-medication as given for the first ECT session. Starting with the third ECT, depressive symptoms gradually resolved.

Temporary arrhythmias and repolarisation abnormalities can occur in cardiologically-healthy subjects and may be physiological side-effects of ECT (Abrams, 1992). However, in our patient such effects were observed only after the combined use of ECT and venlafaxine and not at all after eight ECTs without antidepressants. ECT may enhance central serotonergic responsivity (Shapira et al, 1992). Venlafaxine inhibits both serotonin and noradrenaline uptake. We hypothesise that our patient experienced long-lasting bradycardia because increased serotonin in the central nervous system directly affected brainstem cardiovascular regulation (Shvaloff \& Laguzzi, 1986). Alternatively, unknown interactions between venlafaxine and the anaesthetics used may have caused the adverse effect. Nevertheless, clinicians should note that prolonged bradycardia can complicate the combined use of venlafaxine and ECT.

Abrams, R. (1992) Electroconvulsive Theropy (2nd edn). New York: Oxford University Press.

Shapira, B., Lerer, B., Kindler, S., et al (1992) Enhanced serotonergic responsivity following electroconvulsive therapy in patients with major depression. British journol of Psychiotry. 160, 223-229.

Shvaloff, A. \& Laguzzi, R. (1986) Serotonin receptors in the rat nucleus solitarii and cardiovascular regulation. European Journal of Pharmocology, I32, 283-288.

\section{M. Agelink, T. Zeit, E. Klieser}

Department of Psychiatry, Ev. Hospital

Gelsenkirchen, University of Bochum, Munckelstr.

27. 45879 Gelsenkirchen, Germany

\section{Doxazosin for reboxetine-induced urinary hesitancy}

Sir: Reboxetine is a selective potent noradrenaline uptake inhibitor with clinically documented antidepressant properties. The drug is usually well tolerated; however, especially in elderly male patients, urinary hesitancy and/or retention can be a troublesome side-effect (Berzewski et al, 1997). Recently, we have found that this sideeffect can be mitigated by the co-administration of doxazosin, an $\alpha_{1}$-adrenoceptor antagonist indicated for the treatment of urinary retention associated with prostatism (Dollery, 1991). 
A 59-year-old man was referred to our service in 1997, with a three-year history of depression and obsessive-compulsive syndrome. Previous treatment with sertraline, lofepramine and venlafaxine, administered successively, had little effect. The patient was prescribed fluvoxamine (100 $\mathrm{mg}$ twice daily) which resulted in the alleviation of the obsessive-compulsive syndrome but little improvement in depression. Five months later reboxetine $4 \mathrm{mg}$ twice daily was added; this was followed by gradual lifting of the mood and an increase in the patient's activity level. The patient tolerated the combined medication well, apart from the complaint of urinary hesitancy and the experience of not being able to void his bladder completely. Ten months after referral to our service, a urologist suggested to him that his urinary difficulties may be due to his treatment with reboxetine. The patient discontinued the reboxetine on his own initiative, and informed his psychiatrist about this only two weeks later when his depressive symptoms returned. As the depression worsened, the patient agreed to re-try reboxetine, together with doxazosin $1 \mathrm{mg}$ daily, which was co-prescribed to alleviate the urinary side-effect of reboxetine. Following this intervention, the patient's mood and activity level have improved, without the recurrence of the urinary difficulties.

It is likely that the urinary hesitancy/ retention caused by reboxetine is caused by the potentiation of the effects of sympathetically released noradrenaline in the urinary bladder. Indeed, it is known that sympathetic stimulation, probably via $\alpha_{1}$-adrenoceptor activation, can inhibit the contraction of the detrusor muscle (Anderson, 1993). Doxazosin is a selective $\alpha_{1}$-adrenoceptor antagonist which has been shown to be effective in the treatment of urinary retention. Furthermore, doxazosin does not pass the blood-brain barrier (Dollery, 1991) and thus it would not compromise the therapeutic effectiveness of reboxetine, which is likely to be mediated by the potentiation of central noradrenergic neurotransmission.

Anderson, K.-E. (1993) Pharmacology of lower urinary tract smooth muscles and penile erection tissues. Phormacological Reviews, 45, 253-308.

Berzewski, H., Van Moffeert, M. \& Gagiano, C. A. (1997) Efficacy and tolerability of reboxetine compared with imipramine in a double-blind study in patients. European Neuropsychopharmacology, 7 (suppl. I), S37-S48.

Dollery, C. (1991) Therapeutic Drugs. Edinburgh: Churchill-Livingstone.

E. Szabadi Division of Psychiatry, University of Nottingham, Queen's Medical Centre, Nottingham NG7 $2 \mathrm{UH}$

\section{Physical interventions}

Sir: The use of physical interventions, or restraint, to manage challenging behaviours presented by adults and children with a learning disability is a matter of considerable concern to professionals, care staff, family members and those responsible for developing and implementing Government policy. After the tragic death of Zoe Fairley there can be little doubt of the importance of effective organisational policies and good staff training to ensure that the welfare and safety of service users is not put at risk when they are submitted to physical interventions.

Following the publication of Pbysical Interventions: A Policy Framework (Harris et al, 1996), the British Institute of Learning Disabilities and the National Autistic Society have been funded by the Department of Health to establish an accreditation scheme for courses which teach physical interventions. We would like to hear from anyone who provides training on physical interventions/restraint for staff working with adults and children with a learning disability and/or autism. We should also be pleased to receive comments from any individuals or organisations who have received training on the use of physical interventions.

Harris, J. C., Allen D., Cornick, M., et al (1996) Physical Interventions: A Policy Framework. Kidderminster BILD Publications.

J. Harris British Institute of Learning Disabilities, Wolverhampton Road, Kidderminster, Worcestershire DYI0 3PP

\section{One hundred years ago}

\section{Asylum news}

Derbyshire. - The Committee asked for power to extend the present county asylum at Mickleover so as to provide for 750 patients, as against 600 at present. This would involve an outlay of $£ 21,000$. During the discussion several speakers suggested the desirability of erecting a new asylum in the northern part of the county, which was very favourably regarded by those present.

Lancashire. - At the annual meeting of the Lancashire Asylums Board the Chairman moved the adoption of the Report of the Committee of Winwick Asylum, which stated that the tender of Messrs. Robert Neill and Sons for the erection of the new asylum for Winwick for the sum of $£ 253,000$ had been approved. The patients' blocks will be completed in about two and a half years, and the whole building in three and a half years. The report was confirmed.

West Riding. - In order to meet the need created by an increase of insanity in the West Riding of late years, the Asylums Committee of the County Council are making preparations for the erection of an additional asylum capable of accommodating about 2000 patients. It was shown that whereas in 1887 there were only 2951 patients in the two asylums then open for the receipt of patients (Wakefield and Wadsley), in 1896, ten years later, the three asylums at Wakefield, Wadsley, and Menston contained no fewer than 4152 insane persons. These figures are exclusive of outcounty and private patients. Then, in addition, there were 1060 lunatics in the workhouses of the Riding in 1887, and 928 in 1896 . To put it in another way, the returns for 1887 showed an increase of 105 in the asylums as compared with the previous year, whereas in 1896 an increase of $\mathbf{2 2 6}$ was recorded. If sixteen additional 